\title{
Sentinel lymph node biopsy in breast cancer patients treated with induction chemotherapy should be performed after the completion of chemotherapy
}

\author{
Renata Duchnowska
}

Sentinel lymph node biopsy in patients with early breast cancer treated with breast conserving therapy is currently a standard treatment. SLN biopsy allows to resign from the resection of the axillary lymph nodes and thus, to reduce the risk of complications and to improve the quality of life of the patients. The objective of preoperative chemotherapy is to decrease the scope of surgery both within the primary focus of the tumour and in the axillary lymph nodes. The results of the research carried out so far indicate that SLN biopsy, after a preoperative chemotherapy in patients at the $\mathrm{N}_{0}$ and $\mathrm{N}_{1-2}$ clinical stage is credible and in allows many of them to avoid the resection of axillary lymph nodes. Therefore there are rational arguments justifying SLN biopsy after the completion of preoperative chemotherapy.

NOWOTWORY J Oncol 2017; 67, 4: 270-272

Key words: sentinel lymph node, induction chemotherapy, breast cancer

\section{Introduction}

The sentinel lymph node (SLN) is the first lymph node in the lymphatic drainage of the primary focus of the tumour. It is believed that the SLN is the first site of accumulation of tumour cells at the stage of the occurrence of metastases in the regional lymph nodes [1-3]. The most frequent method of sentinel lymph node identification is preoperative lymphoscin-

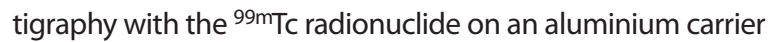
connected with a perioperative staining of lymph drainage with methylene blue. In some centres, in order to increase the sensitivity of SLN identification, hybrid techniques are used i.e. the connection of functional imaging with morphological one (SPECT/CT, single photon emission with a low-dose CT) [4]. During surgery, the lymph nodes with the largest radioactivity and the most stained, which are regarded as SLN are removed. It is admitted that the sensitivity of the SLN biopsy should be above $90 \%$ with the rate of false positive results below $10 \%$. The improvement of the rate of identified SLN is significantly affected by the number of procedures performed by the surgeon, i.e. their experience and practice [5-7].
Preoperative (induction) chemotherapy in breast cancer patients is used in order to decrease the advancement stage of the primary tumour and/or lymph nodes, which allows for a breast conserving procedure. In more advanced cases (T4, N2-3 stage), preoperative chemotherapy may facilitate or make possible a radical surgery procedure [8]. Currently, in patients with so-called aggressive phenotypes, which at the same time are characterised with high sensitivity to chemotherapy and/or molecularly targeted treatment (triple negative cancer and HER2-positive), the induction treatment is also often applied in patients in the NO stage if the tumour's diameter is larger than $2 \mathrm{~cm}[8,9]$. In these phenotypes, the rate of pathologic complete response $(p C R$,$) affects the improvement of survival parameters [9].$

\section{Radiotherapy vs axillary lymphadenectomy in patients in the NO clinical stage and a metastasis in a sentinel lymph node}

In 3 prospective clinical trials (ACOSOG Z0011, AMAROS, IBCSG 23-01) it was shown that in patients with metastases 
in the SLN, the resection of axillary lymph nodes can be avoided. The ACOSOG Z0011 study compared the treatment with and without axillary lymphadenectomy in patients treated with the BCT and post-operative radiotherapy with tangential fields; this led to the conclusion that in the case of metastatic involvement of 1 or 2 SLN, the rate of loco-regional recurrences, the time to progression and the overall survival periods, are similar after 5 years, in spite of the fact that in the group undergoing axillary lymphadenectomy in about $30 \%$ patients, the metastases to consecutive lymph nodes were found [10]. The AMAROS study showed that axillary irradiation is equally effective as surgery with a much lower risk of complications [11]. Similar results were rendered by the IBCSG 23-01 study which concerned patients with micro-metastases in SLN [12].

\section{Preoperative chemotherapy in patients at the NO clinical stage}

Before the commencement of preoperative chemotherapy in patients at the $\mathrm{CNO}$ stage, a number of additional tests must be performed, including an ultrasound of the axilla, connected, when metastases are suspected, with a biopsy of axillary lymph nodes and also to mark the primary tumour with clips or a tattoo. In some centres, in order to evaluate the advancement stage, additionally a breast MRI or position computed tomography (PET/CT) is performed. Postponing the SLN biopsy until the moment of completion of preoperative chemotherapy does not significantly reduce the accuracy of the examination; the rate of identified SLN in this group is more than $90 \%$, and false negative results concern 6-9\% of patients [13-15]. That is why, international guidelines, including those from the most recent conferences on the treatment of early breast cancer - St. Gallen, recommend the SLN biopsy in patients at the N0 stage after the completion of preoperative chemotherapy $[8, w w w$. oncoconferences.ch/BCC].

\section{Preoperative chemotherapy in the patients at the $\mathbf{N}+$ clinical stage}

In the case of diagnosing clinical $\mathrm{cN}+$ stage and its conformation with a fine needle biopsy, there is no need to perform an SLN biopsy before preoperative chemotherapy, as this procedure is performed solely in patients without pathological or clinical symptoms of metastatic involvement of axillary lymph nodes. Regardless of the above, SLN biopsy before preoperative chemotherapy would not change the clinical stage and thus would not affect the choice of the type of surgical intervention, but it would increase the risk of complications and diagnostic costs. It must be remembered that the main objective of preoperative chemotherapy, apart for guaranteeing the possibility of BCT, is to avoid axillary lymphadenectomy. A few studies have shown that the $\mathrm{pCR}$ rate within the lymph nodes after preoperative che-
Table I. pCR rate within the lymph nodes after preoperative chemotherapy

\begin{tabular}{|c|c|c|}
\hline Study & $\mathrm{N}$ & $\begin{array}{l}\text { The rate of pCR in the } \\
\text { lymph nodes }\end{array}$ \\
\hline ACOSOG Z1071 (cT0-4N1/2) ${ }^{16}$ & 649 & $41 \%$ \\
\hline SN FNAC (cT0-3N1/2) ${ }^{17}$ & 145 & $35 \%$ \\
\hline Mamtani (cT0-3N1/2) ${ }^{18}$ & 195 & $49 \%$ \\
\hline
\end{tabular}

motherapy, especially in the following phenotypes: triple-negative and HER2-positive, amounts to 40-50\% [16-18] (Tab. I). What is important, axillary lymphadenectomy may be avoided in about $50 \%$ of patients with SLN biopsy, confirmed with $\mathrm{pCR}$, after pre-operative chemotherapy especially that the majority of them, an account of the baseline metastatic involvement of the axillary lymph nodes, will undergo radiotherapy of the nodal fields [8, 16-18] (www.oncoconferences.ch/BCC). This fits to the generally accepted tendency to limit axillary lymphadenectomies in breast cancer patients [www.oncoconferences.ch/BCC]. What is more, in spite of earlier concerns about SLN identification after preoperative chemotherapy and the high risk of false negative results, a prospective analysis of the ACOSOG Z1071 and SENTINA studies, has shown that the sensitivity of the SLN biopsy in the case of the removal of at least 3 SLNs is larger than $90 \%$, and the rate of false negative results fits in the range of $10 \%[19,20]$.

\section{Summary}

Preoperative chemotherapy is used mostly with the intention of reducing the scope of a surgery, also in relation to the axillary lymph nodes The results of the studies carried out so far show that SLN biopsy after preoperative chemotherapy is a reliable method, allowing, in a large group of patients, to avoid axillary lymphadenectomy. Such a possibility is not guaranteed by SLN biopsy before the commencement of preoperative chemotherapy. As a consequence, currently the SLN biopsy after the completion of chemotherapy is becoming a standard treatment method, irrespective of the baseline status of the axillary lymph nodes [8, 19-21, www. oncoconferences.ch/BCC].

\section{Conflict of interest: none declared}

\section{Renata Duchnowska, MD, PhD}

Department of Oncology

Military Institute of Medicine

Szaserów St. 128, 04-141 Warsaw, Poland

e-mail:rdtt@wp.pl

Received \& Accepted: 7 May 2017

Based on the presentation at the V Annual Conference of the Nowotwory Journal of Oncology, 'Oncological Debates', held in Warszawa, 7-8th April 2017 


\section{References}

1. Barnwell JM, Arredondo MA, Kollmorgen D et al. Sentinel node biopsy in breast cancer. Ann Surg Oncol 1998; 5: 126-130.

2. Veronesi U, Paganelli G, GalimbertiV et al. Sentinel-node biopsy to avoid axillary dissection in breast cancer with clinically negative lymph-nodes. Lancet 1997; 349: 1864-1867.

3. Albertini JJ, Lyman $\mathrm{GH}$, Cox C et al. Lymphatic mapping and sentinel node biopsy in the patient with breast cancer. JAMA 1996; 276: 1818-1822.

4. Chen SL, lddings DM, Scheri RP et al. Lymphatic mapping and sentinel node analysis: current concepts and applications. CA Cancer J Clin 2006; 56: 292-309.

5. Tafra L. The learning curve and sentinel node biopsy. Am J Surg 2001; 182: 347-350.

6. Sanidas EE, de Bree E, Tsiftsis DD. How many cases are enough for accreditation in sentinel lymph node biopsy in breast cancer? Am J Surg 2003; 185: 202-210.

7. Kuehn T, Bembenek A, Decker T et al. Consensus Committee of the German Society of Senology. A concept for the clinical implementation of sentinel lymph node biopsy in patients with breast carcinoma with special regard to quality assurance. Cancer 2005; 103: 451-461.

8. Coates AS, Winer EP, Goldhirsch A et al. Tailoring therapies - improving the management of early breast cancer: St Gallen International Expert Consensus on the Primary Therapy of Early Breast Cancer 2015. Ann Oncol 2015; 26: 1533-1546.

9. Cortazar P, Zhang L, Untch $M$ et al. Pathological complete response and long-term clinical benefit in breast cancer: the $C T N e o B C$ pooled analysis. Lancet 2014; 384: 164-172

10. Giuliano AE, Ballman $K, M c C a l l ~ L$ et al. Locoregional recurrence after sentinel lymph node dissection with or without axillary dissection in patients with sentinel lymph node metastases: Long-term Follow-up From the American College of Surgeons Oncology Group (Alliance) ACOSOG Z0011 Randomized Trial. Ann Surg 2016; 264: 413-420.

11. Donker M, van Tienhoven G, Straver ME et al. Radiotherapy or surgery of the axilla after a positive sentinel node in breast cancer (EORTC 10981-22023 AMAROS): a randomised, multicentre, open-label, phase 3 non-inferiority trial. Lancet Oncol 2014; 15: 1303-1310.
12. Galimberti V, Cole BF, Zurrida S et al. Axillary dissection versus no axillary dissection in patients with sentinel-node micrometastases (IBCSG 23-01): a phase 3 randomised controlled trial. Lancet Oncol 2013; 14: 297-305.

13. Hunt KK, Yi M, Mittendorf EA et al. Sentinel lymph node surgery after neoadjuvant chemotherapy is accurate and reduces the need for axillary dissection in breast cancer patients. Ann Surg 2009; 250: 558-566.

14. Classe JM, Bordes V, Campion L et al. Sentinel lymph node biopsy after neoadjuvant chemotherapy for advanced breast cancer: results of Ganglion Sentinelle et Chimiotherapie Neoadjuvante, a French prospective multicentric study. J Clin Oncol 2009; 27: 726-732.

15. Geng C, Chen X, Pan X, Li J. The feasibility and accuracy of sentinel lymph node biopsy in initially clinically node-negative breast cancer after neoadjuvant chemotherapy: a systemic review and meta-analysis. PLoS One 2016; 11: e0162605.

16. Boughey JC, Suman VJ, Mittendorf EA et al. Sentinel lymph node surgery after neoadjuvant chemotherapy in patients with node-positive breast cancer: the ACOSOG Z1071 (Alliance) clinical trial. JAMA 2013;310:1455-1461.

17. Boileau JF, Poirier B, Basik M et al. Sentinel node biopsy after neoadjuvant chemotherapy in biopsy-proven node-positive breast cancer: the SN FNAC study. J Clin Oncol 2015; 33: 258-264.

18. Mamtani A, Barrio AV, King TA et al. How often does neoadjuvant chemotherapy avoid axillary dissection in patients with histologically confirmed nodal metastases? Results of a Prospective Study. Ann Surg Oncol 2016; 23: 3467-3474.

19. Kuehn T, Bauerfeind I, Fehm T et al. Sentinel-lymph-node biopsy in patients with breast cancer before and after neoadjuvant chemotherapy (SENTINA): a prospective, multicentre cohort study. Lancet Oncol 2013; 14: 609-618.

20. Fu JF, Chen HL, Yang J et al. Feasibility and accuracy of sentinel lymph node biopsy in clinically node-positive breast cancer after neoadjuvant chemotherapy: a meta-analysis. PLoS One 2014; 9: e105316.

21. El Hage Chehade $\mathrm{H}$, Headon $\mathrm{H}$, El Tokhy $\mathrm{O}$ et al. Is sentinel lymph node biopsy a viable alternative to complete axillary dissection following neoadjuvant chemotherapy in women with node-positive breast cancer at diagnosis? An updated meta-analysis involving 3,398 patients. Am J Surg 2016; 212: 969-981. 